\title{
Design of Open Experimental Teaching Management System
}

\author{
Liu Hongqing ${ }^{1}$, Liu Yan ${ }^{2}$ \\ ${ }^{1}$ Hunan Vocational College of Modern Logistics, Changsha, Hunan, 410131, China \\ ${ }^{2}$ Hunan Mechanical \& Electrical Polytechnic, Changsha, Hunan,410151, China
}

Keywords: open type, experimental teaching, management system, design

\begin{abstract}
The system adopts the popular B/S structure and ASP dynamic web page development technology. In data transmission and storage, Microsoft SQL Server 2000 is selected as the platform, and a prototype system of open experimental teaching management is designed and developed. The overall design, development and security measures of the system are introduced in detail, and the system can guide the students to make an experiment appointment according to the actual situation and inquire the results of the experiment.
\end{abstract}

\section{Introduction}

With the rapid development of our school in recent years, the mode of running a school is diversified and the scale of the students is expanding. At this time, it is urgent to study and solve the open type experimental teaching management system with the construction of open laboratory and the use of Internet to establish an open experimental teaching management system. It reform the old teaching model and establish a new system, which is greatly raised. It has increased the utilization rate of laboratories and the enthusiasm of students to do experiments, and relieved managers from heavy laboratory management.

\section{System Design}

The open experimental teaching management system, student experiment management subsystem, is an information system to realize students' online experiments. It is a network based information management system, which provides students with personal information query, password modification, experimental reservation test and successful appointment, the related processing of the reserved items, and the inquiry of experimental results. The student experiment management subsystem, as part of the open experimental teaching management system, can coordinate with the teacher management subsystem and the system management subsystem, so as to achieve the purpose of the experimental teaching management.

Students can login to the system through their username and password, and the system will automatically verify the login information of students. If the information is correct, the student can enter a series of operations in the system of student's permission. After entering the system, the students can carry out their basic information inquiry, experiment reservation test and successful appointment, the processing of the appointment experiment, the inquiry of experimental results, the writing and submission of the experimental report.

(1) access to personal information page, students can query their personal information. But in this page, students can not modify their basic information, and the information can be modified only with their password, and the password is modified with a special page.

(2) enter the password modification page, students can enter the old password, new password and verify the new password, then you can finish the modification of the password. If the old password is wrong, or the new password entered and the new password is inconsistent, it will pop up the wrong information.

(3) after entering the reservation test page, the student should test the subject from the list of questions, and if the test is successful, it will enter the main page of the reservation, otherwise retest or withdraw the reservation. 
(4) after the successful entry to the reservation page, the student should choose the reservation condition, such as: the student chooses the instructor and the time period, then the system will list the appointment information according to the name and time of the instructor.

(5) when students enter their own reservation information page, they cancel the reservation information according to the selected records and their own requirements. If the room has been cancelled by the administrator, the instructor has canceled the experimental project, such as the student himself to cancel the experimental project.

(6) When students want to query their own experimental results, they can make selective inquiries. For example, if a student wants to inquire about the achievements of an experimental project, it will show the results of the experimental project in the list when the name of the experimental project is selected.

\section{Database Design}

Database design refers to a given application environment that provides a logical design for determining the optimal data model and processing, as well as a physical design for determining the storage structure and access methods of the database, which can reflect the information and information connection of the real world, meet the user's data requirements, and be able to be used for a certain data. The library management system is capable of achieving the expected functions and effectively storing the required data. The database has become the core of the modern information society. Under the support of the database management system, a series of operations such as collecting, sorting, storing, retrieving and updating information are carried out. For database developers, designing their databases must consider the quality of the design, which directly affects the efficiency and quality of the entire system, which will lead to the success of the system development.

\subsection{Entity integrity}

Entity integrity refers to the integrity of rows in a table, requiring all rows in the table to have a unique identifier called primary key. Whether the primary key can be modified or whether the entire column can be deleted depends on the completeness required between the primary key and other tables. Its rules mainly mean that the primary key can not be empty. For example, in the student information table, the student number is the primary key, indicating that it is impossible for two students who have the same student number to represent the uniqueness.

\subsection{Value integrity}

Value integrity refers to the integrity of the range of the columns. Such as data type, format, range range, whether to allow null values, etc. Value integrity restricts the values appearing in some attributes and restricts them to a finite set.

\subsection{Reference integrity}

Referential integrity is an important concept in database design. Referential integrity refers to the consistency between the two key and the foreign key data. It ensures the row of the foreign key corresponding to other tables in the table with the primary key. In SQL Server, referential integrity is shown in the following aspects.

It is forbidden to insert data rows containing key words that are not found in the main table from the table.

Forbidden will cause the corresponding value from the table to change the outside key value in the isolated main table.

It is forbidden to delete the main table records with corresponding records in the table.

Referential integrity is the constraint between two tables.

For example, in the reservation information table, the student number is a foreign key, which is used to contact the student information table when the student's reservation information is querying. 


\subsection{User custom integrity}

According to the different application environment, the relational database system often needs some special constraints. The integrity of the user defined is the constraint conditions for a specific application field and the relational database.

\section{System Test}

System testing is a very important and long - term stage in the process of system development. The purpose is to ensure the quality of the system development and the reliability and stability of the system. The whole design and implementation process of the system has gone through the research, requirement analysis, database design and code compilation, and finally entered the testing stage. And what we need to do in the process of testing is to correct some shortcomings and defects in the system, and make the system perfect. And if the system test this link has not done properly, it will make the development of the system come to naught.

\subsection{Test principles}

The test of the system should be carried out by a lot of people, and in the test stage of the actual application development, the specialized test personnel should be tested by the special testers, and the actual development of the teaching management system is taken into account. Therefore, the test stage is tested by the teachers or students after many experiments.

In the process of testing, we must consider the possible execution situations as far as possible. And in the test stage, we can not only input some reasonable data, but also use some unreasonable data to test, so that it is easy to deal with the unreasonable abnormality.

\subsection{Test method}

There are two ways to test any system product: black box testing and white box testing. Black box testing means that after knowing the functions that should be provided, it is tested whether every function can be used normally. The white box test is aware of the internal working process of the system, and can test whether the internal work process is carried out according to the regulations

\section{Summary}

With the deepening of teaching reform in Colleges and universities, the development of experimental teaching management system has become inevitable. In the course of developing this topic, based on the current situation of laboratory, we choose ASP technology. The whole design process of the system has been completed and the function is basically realized, especially in the design of the student reservation module, and the possible situations that may occur in the actual process are considered.

\section{References}

[1] Stephygraph, L.R., Arunkumar, N. Brain-actuated wireless mobile robot control through an adaptive human-machine interface (2016) Advances in Intelligent Systems and Computing, 397, pp. 537-549.

[2] Weisen Pan, Shizhan Chen, Zhiyong Feng. Investigating the Collaborative Intention and Semantic Structure among Co-occurring Tags using Graph Theory. 2012 International Enterprise Distributed Object Computing Conference, IEEE, Beijing, pp. 190-195.

[3] Arunkumar, N., Jayalalitha, S., Dinesh, S., Venugopal, A., Sekar, D. Sample entropy based ayurvedic pulse diagnosis for diabetics (2012) IEEE-International Conference on Advances in Engineering, Science and Management, ICAESM-2012, art. no. 6215973, pp. 61-62.

[4] Arunkumar, N., Ramkumar, K., Hema, S., Nithya, A., Prakash, P., Kirthika, V. Fuzzy Lyapunov exponent based onset detection of the epileptic seizures (2013) 2013 IEEE Conference on 
Information and Communication Technologies, ICT 2013, art. no. 6558185, pp. 701-706.

[5] Jonathan J. Faig, Alysha Moretti, Laurie B. Joseph, Yingyue Zhang, Mary Joy Nova, Kervin Smith, and Kathryn E. Uhrich, Biodegradable Kojic Acid-Based Polymers: Controlled Delivery of Bioactives for Melanogenesis Inhibition, Biomacromolecules, 2017, 18(2), 363-373. 